\title{
Análisis de la satisfacción del alumnado de L2 con respecto a la adquisición de terminología especializada por medio de videojuegos: estudio de caso
}

\author{
José Ramón CALVO-FerRer \\ UCAM Universidad Católica de Murcia \\ José RAMÓN BELDA-MEDINA \\ Universidad de Alicante
}

Recibido: 1 marzo 2014 / Aceptado: 11 enero 2015

ISSN: $1697-7467$

\begin{abstract}
RESUMEN: Los videojuegos permiten enseñar contenidos y destrezas de forma eficiente, posibilitando un aprendizaje duradero (Rama et al., 2012), y aumentan la motivación y la implicación del alumnado (Martens et al., 2004). En esta línea, el presente estudio pretende medir tanto el grado de satisfacción de dos grupos de estudiantes de L2 de la Universidad de Alicante con respecto a la adquisición de terminología especializada por medio de un videojuego como la percepción sobre el propio grado de aprendizaje. Tras un período de práctica, se ha medido y analizado tanto el grado de aprendizaje alcanzado como la satisfacción con la herramienta empleada y, muy especialmente, las diferencias en el grado de aprendizaje percibido por cada uno de estos grupos.
\end{abstract}

Palabras clave: terminología, adquisición, vocabulario, videojuegos, satisfacción

\section{Students' Satisfaction in L2 Terminology Acquisition with Video Games: A Case Study}

\begin{abstract}
Video games are believed to efficiently teach contents and skills, allowing long-lasting learning (Rama et al., 2012) and to increase students' motivation and engagement (Martens et al., 2004). This study aims to measure both the degree of student satisfaction in L2 terminology acquisition through a video game and how it affects the way L2 students from the University of Alicante perceive such learning outcomes. Following a period of practice, their performance in specialised lexis in English, the degree and satisfaction with the learning tool provided and their perceived learning outcomes in terminology acquisition has been measured and subsequently analysed.
\end{abstract}

Keywords: terminology, acquisition, vocabulary, video games, satisfaction

\section{InTRODUCCIÓN}

La mayor parte del alumnado se maneja con soltura en el lenguaje de los videojuegos. Los videojuegos tienen la capacidad no sólo de enseñar contenidos y capacidades, sino de hacerlo de forma más eficiente, dando pie a un aprendizaje duradero (Rama, Black, van Es \& Warschauer, 2012). Distintos estudios indican que los videojuegos ofrecen al jugador 
una completa inmersión en cualquier tipo de actividad, lo que les convierte en herramientas óptimas para el aprendizaje (Schrader \& McCreery, 2008). El contexto sociocultural que reproducen y el feedback inmediato que ofrecen, junto con la diversión que proporcionan al jugador, los hace especialmente útiles en el ámbito de las lenguas extranjeras en general y en el desarrollo de las competencias del intérprete en particular.

De igual manera, distintos estudios afirman que los videojuegos aumentan la motivación del estudiante y su implicación en la materia de estudio (Martens et al. 2004). Específicamente en el ámbito de la traducción e interpretación, los videojuegos facilitan la mejora de la competencia terminológica en lengua extranjera (Calvo-Ferrer, 2013). En línea con ello, el presente estudio pretende mostrar el grado de satisfacción del alumnado de la asignatura Lengua B(III): Inglés del Grado en Traducción e Interpretación de la Universidad de Alicante con el videojuego The Conference Interpreter frente a otras herramientas tradicionales para la adquisición de terminología especializada en segundas lenguas.

De este modo, se ofrece a continuación un breve recorrido por los videojuegos como herramienta para la formación y la educación, prestando especial atención a los resultados investigadores obtenidos en el ámbito del aprendizaje de lenguas extranjeras. Tras ello, se realiza una breve descripción del objeto de estudio: el nivel de satisfacción, tal como se entiende en el marco de la presente investigación, así como de los materiales empleados para su medición y las herramientas empleadas por el alumnado durante el proceso de aprendizaje. Finalmente, se ofrece un análisis de los resultados obtenidos por medio de tales herramientas y se presentan las conclusiones que se derivan de los mismos.

\section{VIDEOJUEgOS PARA LA EDUCACIÓN Y LA FORMACIÓN}

Partiendo de su carácter motivador, son varios los autores que hacen referencia a los videojuegos como herramientas beneficiosas para el aprendizaje. Kierriemuir y McFarlane (2004) señalan que los videojuegos son materiales interactivos con capacidad para atraer la atención del alumno, mientras que Gee (2003) asegura que, con un seguimiento del profesorado, los videojuegos pueden ayudar a adquirir los conocimientos y el lenguaje de profesionales de distintos dominios semióticos. Moreno-Ger, Burgos y Torrente (2009) exponen que, dado el atractivo que poseen ante los ojos del alumnado, los videojuegos pueden ser una herramienta muy útil para el aprendizaje, puesto que la motivación es un factor clave para aprender de forma efectiva (Cordova \& Lepper, 1996).

En esta línea, estudios como el de Ricci et al. (1996) ofrecen resultados positivos no sólo al respecto de aprendizaje y memorización, sino acerca del grado de satisfacción derivado del empleo de los videojuegos. Los videojuegos modernos favorecen una completa inmersión del jugador en la actividad, permitiendo que éste sea quien decide en parte el desarrollo de la misma, favorecen la competitividad y los entornos colaborativos, ofreciendo además un entorno de práctica seguro en el que el jugador obtiene un feedback inmediato respecto a sus acciones y favoreciendo un aprendizaje por medio de ensayo y error, características deseables en un entorno de aprendizaje constructivista y centrado en el estudiante (de Freitas \& Oliver, 2006).

Numerosas investigaciones han dado cuenta de la efectividad de los videojuegos como herramienta para el aprendizaje en áreas del conocimiento tales como álgebra (Mayo, 2007), 
fisiología (Wong et al., 2007), conocimiento del medio (Barab, Thomas, Dodge, Carteaux \& Tuzun, 2005), ingeniería (Coller \& Scott, 2009), negocios y gestión empresarial (Blunt, 2009), comunicación intercultural en el ámbito de los negocios (Guillén-Nieto \& AlesonCarbonell, 2012), urbanismo (Poplin, 2012) o medicina (Göbel, Hardy, Wendel, Mehm \& Steinmetz, 2010).

En referencia al aprendizaje de idiomas extranjeros, Schwienhorst (2002) afirma que los videojuegos ofrecen un contexto sociocultural realista que favorece el aprendizaje de segundas lenguas, mientras que Nesselhauf y Tschichold (2002) aseguran que la implementación en el aula y el aprendizaje de ejercicios de colocaciones en lengua inglesa con la ayuda de videojuegos ayuda a mejorar el vocabulario. Hu y Chang (2008) afirman que los videojuegos ofrecen un enorme potencial para mejorar la comprensión auditiva de los estudiantes de inglés como lengua extranjera, mientras que Huyen y Nga (2003) concluyen que los juegos digitales son una herramienta óptima para mejorar las competencias comunicativas de los estudiantes. Por su parte, Miller y Hegelheimer (2006) afirman que aquellos alumnos que juegan al videojuego The Sims mejoran de forma significativa sus conocimientos respecto a gramática y vocabulario en lengua inglesa.

Rankin, Gold y Gooch (2006) opinan que el aprendizaje que se produce en el mundo virtual puede ser traspasado al mundo real, dado que los videojuegos promueven el desarrollo de destrezas comunicativas y favorecen la experimentación del alumno en un entorno virtual por medio de los sentidos y de la imaginación, propiciando una interacción social entre jugadores, lo que mejora su vocabulario en lengua inglesa. Anderson et al. (2008) afirman que los videojuegos preparan a los estudiantes para interactuar en sociedad permitiendo la creación de vínculos naturales entre jugadores de distintas nacionalidades, posibilitando en última instancia el aprendizaje de la lengua inglesa como idioma extranjero. Yip y Kwan (2006) aseguran que los videojuegos son herramientas útiles para el aprendizaje de vocabulario en lengua extranjera, que los alumnos suelen preferir juegos online como herramienta docente frente a la metodología tradicional presencial y que para mantener el interés del alumnado y garantizar la efectividad de la enseñanza se antoja necesario el diseño de juegos más motivadores que permitan a los estudiantes alcanzar distintos objetivos y les ofrezcan una visión más amplia y contextualizada de su aprendizaje.

\section{ObJetivos de LA PRESENTE INVESTigación}

La presente investigación parte de la premisa de que los videojuegos, en cuanto herramientas eminentemente prácticas, generan aprendizaje. Por tanto, el objetivo de la misma es analizar si este procedimiento genera un mayor grado de satisfacción en el estudiante, tal como sugieren estudios previos como el de Lai-Chong Law et al. (2008), en comparación con otras herramientas tradicionales.

En el marco de la presente investigación se entiende el concepto de satisfacción como la adecuación del tratamiento aplicado a los sujetos participantes en la experimentación a su gusto y expectativas, interpretándose en la práctica como la confluencia de dos aspectos fundamentales: la satisfacción respecto a la herramienta empleada y la satisfacción respecto al propio aprendizaje. 
De este modo, en primer lugar, se ha tomado en consideración la opinión de los sujetos participantes respecto a la propia herramienta empleada en relación con la percepción de los sujetos respecto a la utilidad y el carácter motivador de las herramientas puestas a su disposición. En segundo lugar, se ha pretendido recabar de los sujetos participantes información al respecto de la propia percepción sobre el aprendizaje resultante, esto es, el grado en que el empleo de las distintas herramientas ha favorecido el desarrollo de las competencias lingüísticas puestas en práctica durante la experimentación.

\section{Diseño experimental}

El diseño experimental consta de distintas fases, conducentes a la comprobación de la hipótesis planteada y al análisis y discusión de los resultados obtenidos. En primer lugar, la asignación al azar de los alumnos de la asignatura Lengua B(III): Inglés en dos grupos equivalentes: un grupo de control y otro experimental. Posteriormente, la observación o medición de la competencia terminológica en lengua inglesa sobre sistemas operativos móviles por medio de un pre-test, idéntico para ambos grupos.

Tras ello, y durante tres sesiones diarias de dos horas, la aplicación de tratamientos distintos a cada uno de los grupos. El grupo de control ha realizado una serie de ejercicios de comprensión auditiva de opción múltiple relacionados con sistemas operativos móviles, marcando las respuestas correctas en un cuadernillo, mientras que el grupo experimental ha tenido acceso a los mismos materiales por medio del videojuego The Conference Interpreter, sin que haya habido diferencia alguna en los contenidos didácticos puestos a disposición de ambos grupos. Es decir, tales materiales didácticos, que son la esencia del tratamiento aplicado a cada uno de los distintos grupos, se han desarrollado a partir de una serie de materiales reales y han sido aplicados a ambos grupos de forma contemporánea y en idénticas condiciones durante tres sesiones de dos horas. Con posterioridad al tratamiento, se ha llevado a cabo una nueva observación tanto del grupo experimental como del de control, por medio de un post-test. Finalmente, al cabo de seis semanas, se ha realizado una nueva observación a través de un test diferido o seguimiento.

Mientras que los resultados relativos a la mejora de la competencia terminológica han sido recogidos en pre-test, post-test y seguimiento y analizados en trabajos anteriores (Calvo-Ferrer, 2013), la presente investigación analiza los datos recogidos en el momento post-test $\left(\mathrm{O}_{1}, \mathrm{O}_{4}\right)$, posterior a la aplicación del tratamiento $\left(\mathrm{X}_{1}, \mathrm{X}_{2}\right)$, en relación exclusiva con el grado de satisfacción del alumnado participante en la experimentación.

\section{Herramientas empleadas}

Tras la aplicación del tratamiento, se ha solicitado a los sujetos participantes la cumplimentación de un cuestionario para determinar el nivel de satisfacción con el tratamiento recibido, así como el grado de motivación e interés que éste les ha despertado. Dicho cuestionario contiene, por una parte, distintas preguntas de respuesta múltiple para que los sujetos participantes en la experimentación valoren, en una escala de 1 a 5 , varios aspectos relacionados con el nivel de satisfacción con el tratamiento, con la dificultad o facilidad de los materiales y con la propia percepción del grado de adquisición de competencias. 
En línea con el objeto de la presente investigación, el cuestionario de satisfacción ha sido diseñado para identificar posibles diferencias en la satisfacción de ambos grupos con relación a la herramienta empleada durante la intervención, es decir, para determinar el grado de satisfacción de los sujetos participantes con el videojuego The Conference Interpreter y las actividades de comprensión auditiva de opción múltiple en cuanto herramientas para la adquisición de competencias del grado en Traducción e Interpretación.

En lo relativo a las preguntas que componen el cuestionario, éstas recogen el dualismo sobre el que se articula el concepto de satisfacción. De este modo, el cuestionario contiene preguntas relativas al propio aprendizaje y respecto a la herramienta empleada, tal como se detalla en el siguiente apartado dedicado a los resultados obtenidos.

\section{Resultados obtenidos}

A continuación se presenta detalle de las respuestas ofrecidas por el alumnado a cada una de las preguntas del cuestionario, analizando las diferencias existentes entre el grupo experimental y el grupo de control, tanto para la totalidad del cuestionario en general, como para cada una de las preguntas en particular. La variable numérica «satisfacción» se ha calculado a partir de 17 preguntas a las que el alumno ha respondido seleccionando un valor numérico, en consonancia con su opinión: «Valora las siguientes afirmaciones (5: totalmente de acuerdo, 1: totalmente en desacuerdo)».

\subsection{Preguntas relativas a la satisfacción del alumnado respecto al propio aprendizaje}

A partir de los datos obtenidos mediante el cuestionario completado por el alumnado a la conclusión del período de intervención se han evaluado las diferencias existentes entre un grupo y otro con respecto a la satisfacción con el grado de aprendizaje alcanzado por medio de la herramienta empleada. De este modo, la tabla 1 recoge la diferencia de medias entre grupos, mientras que en la tabla 2 se presenta el correspondiente ANOVA.

A la luz de los resultados obtenidos, se puede afirmar que existen diferencias estadísticamente significativas entre ambos grupos $[F(1,57)=12.289, \mathrm{p}=.001]$, siendo el grado de satisfacción con el grado de aprendizaje alcanzado mayor en el grupo experimental. Dicho con otras palabras, el alumnado percibe de mayor utilidad como herramienta para la educación y la formación el videojuego The Conference Interpreter que los ejercicios de comprensión auditiva de opción múltiple.

Tabla 1. Satisfacción con el grado de aprendizaje alcanzado en ambos grupos. Estadísticos descriptivos

\begin{tabular}{|c|c|c|c|}
\hline Group & Mean & Std. Deviation & N \\
\hline Experimental & 3.6606 & .36979 & 32 \\
\hline Control & 3.2741 & .47677 & 27 \\
\hline Total & 3.4837 & .46121 & 59 \\
\hline
\end{tabular}


Del mismo modo, el análisis realizado ofrece una potencia muy alta [est pow $=.931$ ], lo cual garantiza la posibilidad de detectar cualquier efecto presente. En esta línea, el estadístico eta cuadrado parcial observado [est $\eta^{2}=.177$ ] indica que, de la varianza de la «satisfacción con el aprendizaje alcanzado», el efecto que se debe al grupo es del 17,7\%. Por tanto, cabe afirmar que, también a este respecto, existe una mayor satisfacción, estadísticamente significativa, en el grupo del videojuego que en el que ha empleado una herramienta tradicional.

Tabla 2. Satisfacción con el grado de aprendizaje alcanzado.

Prueba de los efectos inter-sujetos

\begin{tabular}{|c|c|c|c|c|c|c|c|c|}
\hline Source & $\begin{array}{c}\text { Type III } \\
\text { Sum of } \\
\text { Squares }\end{array}$ & df & $\begin{array}{c}\text { Mean } \\
\text { Square }\end{array}$ & F & Sig. & $\begin{array}{c}\text { Partial Eta } \\
\text { Squared }\end{array}$ & $\begin{array}{c}\text { Noncent. } \\
\text { Parameter }\end{array}$ & $\begin{array}{c}\text { Observed } \\
\text { Power }^{b}\end{array}$ \\
\hline $\begin{array}{c}\text { Corrected } \\
\text { Model }\end{array}$ & $2.188^{\mathrm{a}}$ & 1 & 2.188 & 12.289 & .001 & .177 & 12.289 & .931 \\
\hline Intercept & 704.234 & 1 & 704.234 & 3955.108 & .000 & .986 & 3955.108 & 1.000 \\
\hline GROUP & 2.188 & 1 & 2.188 & 12.289 & .001 & .177 & 12.289 & .931 \\
\hline Error & 10.149 & 57 & .178 & & & & & \\
\hline Total & 728.383 & 59 & & & & & & \\
\hline $\begin{array}{l}\text { Corrected } \\
\text { Total }\end{array}$ & 12.337 & 58 & & & & & & \\
\hline \\
a. R Squared $=.177$ Adjusted R Squared =.163) \\
\hline
\end{tabular}

Por otra parte, se ha desarrollado un análisis en términos porcentuales, tal como se detalla a continuación, como complemento a este análisis estadístico. Con respecto a las preguntas relativas al grado de satisfacción respecto al propio aprendizaje, tanto grupo experimental como grupo de control muestran cierta tendencia generalizada a valorar positivamente los resultados de aprendizaje obtenidos tras la aplicación del tratamiento. De este modo, hallamos que la media aritmética del grupo experimental es de 3,66 (sobre 5,00), mientras que la del grupo de control es de 3,28. No obstante, no todas las preguntas que componen este bloque obtienen los mismos resultados en grupo experimental y grupo de control, por lo que se antoja oportuno realizar un análisis detallado de cada una de ellas, realizando una comparativa de cada uno de los grupos y atendiendo además a cuestiones relativas a media, mediana y moda.

Pregunta 1: «He mejorado mi comprensión auditiva». Con respecto a la primera pregunta del cuestionario, la media para el grupo experimental es del 4,47, mientras que la del grupo de control es 4,15, lo que muestra un alto grado de aceptación por parte de ambos grupos del supuesto.

Pregunta 2: «He mejorado mis conocimientos de gramática». Por lo que respecta a la percepción del alumnado acerca de la mejora de sus conocimientos gramaticales, la media 
es inferior en el grupo de control (2,33), siendo la respuesta más ofrecida el $2(59,26 \%$ de las respuestas), mientras que para el grupo experimental la media es del 2,69 y la moda un $3(50,00 \%$ de las respuestas).

Pregunta 3: «He mejorado mis conocimientos de terminología especializada». La percepción acerca de la adquisición de terminología especializada es superior en el grupo experimental (4,50 de media) frente al grupo de control $(3,89$ de media), lo que indica que, si bien ambos grupos perciben que el tratamiento recibido les ha permitido mejorar sus conocimientos terminológicos, esta percepción es notablemente mayor entre los sujetos que han jugado con el videojuego The Conference Interpreter durante el desarrollo de la experimentación.

Pregunta 4: «He mejorado mis conocimientos sobre los contenidos de los materiales». Tanto mediana como moda se corresponde con un cuatro en ambos grupos, lo que muestra un grado de aceptación similar por parte de ambos grupos respecto al supuesto planteado por la pregunta, si bien la media para el grupo experimental es del 4,03, mientras que la del grupo de control es 3,56, un tanto inferior.

Pregunta 5: «Soy capaz de reproducir la información contenida en los materiales». Tanto grupo experimental como grupo de control ofrecen respuestas similares: mediana y moda se sitúan en un 3 , si bien la media es levemente favorable al grupo experimental $(3,38)$ frente al grupo de control $(3,04)$.

Pregunta 6: «Soy capaz de traducir terminología especializada sobre sistemas operativos móviles en lengua inglesa al español». Se observa que el videojuego The Conference Interpreter potencia esta idea: las medias para grupo experimental y grupo de control son del 3,53 y 3,15, respectivamente, mientras que tanto mediana como moda son también más elevadas en el grupo experimental $(4,00)$, frente al grupo de control $(3,00)$.

Pregunta 7: «Soy capaz de traducir terminología especializada sobre sistemas operativos móviles en lengua española al inglés». Tanto mediana como moda se sitúan en un 3, si bien nuevamente la media es levemente favorable al grupo experimental $(3,03)$ frente al grupo de control $(2,81)$.

\subsection{Preguntas relativas a la satisfacción del alumnado respecto a la herramienta empleada}

Tras llevar a cabo la comparativa entre el grado de satisfacción respecto al grado de aprendizaje, se ha pasado a evaluar el grado de satisfacción del alumnado con la herramienta empleada. De este modo, la diferencia de las medias queda reflejada en la tabla 3 , mientras que en la tabla 4 se presenta el correspondiente ANOVA.

Tabla 3. Satisfacción con la herramienta empleada en ambos grupos Estadísticos descriptivos

\begin{tabular}{|c|c|c|c|}
\hline Group & Mean & Std. Deviation & N \\
\hline Experimental & 4.0031 & .54446 & 32 \\
\hline Control & 2.8074 & .73324 & 27 \\
\hline Total & 3.4559 & .87203 & 59 \\
\hline
\end{tabular}


Complementario a este análisis estadístico, se ha llevado a cabo un análisis en términos porcentuales. Con respecto al segundo bloque de preguntas del cuestionario, es decir, aquellas relativas al grado de satisfacción del alumnado respecto a la herramienta empleada, encontramos, contrariamente a las preguntas del primer bloque, una marcada tendencia por parte de los sujetos del grupo experimental a valorar de forma más positiva la herramienta empleada durante la aplicación del tratamiento que el grupo de control: de este modo, la media aritmética del grupo experimental para la totalidad de las preguntas relativas a la herramienta empleada es de 4,00 (sobre 5,00), mientras que la del grupo de control es de 2,81, lo que evidencia una clara preferencia por el videojuego The Conference Interpreter frente a los listenings puestos a disposición del grupo de control.

Tabla 4. Satisfacción con la herramienta empleada. Prueba de los efectos inter-sujetos

\begin{tabular}{|c|c|c|c|c|c|c|c|c|}
\hline Source & $\begin{array}{l}\text { Type III } \\
\text { Sum of } \\
\text { Squares }\end{array}$ & $\mathrm{df}$ & $\begin{array}{l}\text { Mean } \\
\text { Square }\end{array}$ & $\mathrm{F}$ & Sig. & $\begin{array}{c}\text { Partial Eta } \\
\text { Squared }\end{array}$ & $\begin{array}{l}\text { Noncent. } \\
\text { Parameter }\end{array}$ & $\begin{array}{c}\text { Observed } \\
\text { Power }^{\mathrm{b}}\end{array}$ \\
\hline $\begin{array}{c}\text { Corrected } \\
\text { Model }\end{array}$ & $20.937^{a}$ & 1 & 20.937 & 51.511 & .000 & .475 & 51.511 & 1.000 \\
\hline Intercept & 679.241 & 1 & 679.241 & 1671.115 & .000 & .967 & 1671.115 & 1.000 \\
\hline GROUP & 20.937 & 1 & 20.937 & 51.511 & .000 & .475 & 51.511 & 1.000 \\
\hline Error & 23.168 & 57 & .406 & & & & & \\
\hline Total & 748.770 & 59 & & & & & & \\
\hline $\begin{array}{c}\text { Corrected } \\
\text { Total }\end{array}$ & 44.105 & 58 & & & & & & \\
\hline \multicolumn{9}{|c|}{ a. $\mathrm{R}$ Squared $=.475($ Adjusted R Squared $=.465)$} \\
\hline b. Comp & ed using & & & & & & & \\
\hline
\end{tabular}

Con todo, como sucede con relación al primer bloque, no todas las preguntas relacionadas con la percepción de los sujetos respecto a la utilidad, motivación, e idoneidad de ambos tratamientos como herramienta para la formación y el aprendizaje obtienen resultados similares, por lo que se antoja igualmente oportuno llevar a cabo un análisis detallado de cada una de ellas, realizando una comparativa de cada uno de los grupos y atendiendo, del mismo modo, a cuestiones relativas a media, mediana y moda.

Pregunta 8: «La herramienta de trabajo me ha parecido útil». El grupo experimental se muestra de acuerdo con la afirmación planteada $(4,22$ de media, sobre 5,00$)$ en mayor medida que el grupo de control (3,11 de media). Del mismo modo, tanto mediana como moda son superiores en el grupo experimental (4,00 y 5,00 respectivamente) que en el grupo de control (3,00 y 4,00 respectivamente). De ello se deduce que, en opinión de los sujetos participantes, el videojuego The Conference Interpreter es de mayor utilidad como herramienta para la educación y la formación que los listenings puestos a disposición del grupo de control. 
Pregunta 9: «La herramienta de trabajo me ha parecido motivadora». A tenor de las respuestas recogidas, el grupo experimental (4,19 de media) considera la herramienta empleada más motivadora que el grupo de control (2,30 de media). En esta línea, tanto mediana como moda son superiores en el grupo de experimental (4,00 y 5,00 respectivamente) que en el grupo de control (2,00 para ambos valores).

Pregunta 10: «He disfrutado con la herramienta de trabajo». Mientras que el grupo experimental considera que ha disfrutado con la herramienta empleada (4,00 de media), el grupo de control valora este grado de diversión de forma menos positiva (2,44 de media). Tanto mediana como moda son superiores en el grupo experimental $(4,00$ para ambos valores) que en el grupo de control ( 2 y 3, respectivamente), de lo que se deduce que los sujetos participantes han disfrutado más con el videojuego The Conference Interpreter que con los listenings.

Pregunta 11: «Considero la herramienta empleada adecuada para mejorar mi comprensión auditiva en lengua inglesa». El grupo experimental (4,63 de media) muestra un mayor grado de conformidad con esta afirmación que el grupo de control $(3,81)$, y tanto mediana como moda son superiores en el grupo experimental (5 para ambos valores) que en el grupo de control (4,00 para ambos valores), de lo que se deduce que los sujetos participantes consideran el videojuego The Conference Interpreter una herramienta más adecuada para la mejora de la comprensión auditiva en lengua inglesa que los listenings puestos a disposición del grupo de control.

Pregunta 12: «Considero la herramienta empleada adecuada para mejorar mis conocimientos de gramática en lengua inglesa». La media de las respuestas ofrecidas por el grupo experimental $(2,72)$ es superior a la del grupo de control $(2,22)$ y tanto mediana como moda son superiores en el grupo experimental (3,00 para ambos valores) que en el grupo de control (2,00 para ambos valores), de lo que se deduce que los sujetos participantes perciben un mayor aprendizaje en cuestiones relativas a gramática inglesa a través del videojuego The Conference Interpreter que con los listenings.

Pregunta 13: «Considero que la herramienta empleada me ayuda a mejorar mis destrezas como traductor». Se observa que ambos grupos muestran su conformidad con la afirmación, si bien el grupo experimental (4,09 de media) muestra un mayor grado de conformidad con la misma que el grupo de control $(3,37)$. En esta línea, tanto mediana como moda son equivalentes tanto en el grupo experimental como en el grupo de control (4,00 para ambos valores y grupos).

Pregunta 14: «Considero que la herramienta empleada me ayuda a mejorar mis destrezas como intérprete». Ambos grupos valoran positivamente esta afirmación, si bien el grupo experimental (4,44 de media) lo hace en mayor medida que el grupo de control $(3,59$ de media). Tanto mediana como moda son superiores en el grupo experimental (5,00 para ambos valores) que en el grupo de control (4,00 para ambos valores), de lo que se deduce que los sujetos participantes consideran el videojuego The Conference Interpreter una herramienta más adecuada para la mejora de las destrezas relacionadas con la interpretación que los listenings puestos a disposición del grupo de control.

Pregunta 15: «Considero que el feedback recibido durante el proceso de aprendizaje ha sido adecuado». El grupo experimental se muestra de acuerdo con la afirmación planteada (3,84 de media) en mayor medida que el grupo de control (2,22 de media). Del mismo modo, tanto mediana como moda son sensiblemente superiores en el grupo experimental 
$(4,00)$ que en el grupo de control (2,00 y 1,00 respectivamente). De ello se deduce que el videojuego The Conference Interpreter ofrece un feedback satisfactorio.

Pregunta 16: «Estoy satisfecho con la herramienta empleada». Nuevamente, existe una diferencia considerable entre la media ofrecida por el grupo experimental $(4,25)$ y el grupo de control $(2,70)$, así como en la mediana y la moda tanto de grupo experimental $(4,00 \mathrm{y}$ 5,00 respectivamente) como de grupo de control (3,00 y 2,00 respectivamente). De ello se desprende que el videojuego produce una mayor satisfacción en el marco de la presente investigación que los ejercicios de comprensión auditiva de opción múltiple.

Pregunta 17: «Me gustaría poder estudiar mis asignaturas por medio de la herramienta empleada». De igual manera, el grupo experimental muestra mayor conformidad con la última de las afirmaciones (3,66 de media) que el grupo de control (2,30 de media), lo cual incide en el carácter atractivo del videojuego como herramienta para la educación y la formación a ojos del alumnado.

\section{Conclusiones}

Las distintas pruebas estadísticas realizadas demuestran que el nivel de satisfacción respecto a la utilidad y el carácter motivador con la herramienta empleada es superior en el grupo que ha empleado el videojuego The Conference Interpreter $\left(\mathrm{M}_{\mathrm{GE}}=4,00\right)$ que en el grupo que ha realizado los ejercicios de listening $\left(\mathrm{M}_{\mathrm{GC}}=2,80\right)$. Los resultados obtenidos en la experimentación corroboran que el videojuego es considerado una herramienta más útil y motivadora para la educación y la formación que los ejercicios de comprensión auditiva de opción múltiple. De igual manera, el análisis detallado en términos porcentuales de cada una de las preguntas del cuestionario que hacen referencia a la satisfacción con la herramienta empleada pone de manifiesto que el videojuego The Conference Interpreter resulta una herramienta más atractiva para el alumnado.

Por otra parte, los resultados de las distintas pruebas estadísticas confirman además que la satisfacción con los resultados de aprendizaje obtenidos es superior en el grupo que ha utilizado el videojuego $\left(\mathrm{M}_{\mathrm{GE}}=3,66\right)$ con respecto al grupo que ha accedido a los contenidos didácticos por medio de ejercicios de comprensión auditiva de opción múltiple $\left(\mathrm{M}_{\mathrm{GC}}=\right.$ 3,27). Del análisis detallado de cada uno de los ítems del cuestionario resulta especialmente notable la satisfacción en la mejora de la comprensión auditiva y de las destrezas del intérprete en general. Cabe recordar nuevamente que tanto los materiales que conforman ambos tratamientos como el tiempo y condiciones de exposición a los mismos han sido idénticos en cada uno de los grupos, por lo que tales diferencias han de ser obligatoriamente atribuidas a la herramienta empleada por cada uno de los grupos.

Teniendo en cuenta que la principal diferencia entre tales herramientas radica en el carácter inmersivo y el feedback que ofrece el videojuego The Conference Interpreter frente a los ejercicios de comprensión auditiva, podemos afirmar que tanto el carácter inmersivo como el feedback inmediato son factores determinantes que, en igualdad de condiciones de aplicación, hacen de los videojuegos herramientas que ofrecen más satisfacción al usuario frente a otras herramientas tradicionales no sólo en relación con la motivación por la materia de estudio, sino respecto a la percepción del grado de aprendizaje alcanzado por parte del usuario. 


\section{Bibliografía}

Anderson, T., Reynolds, B. L., Yeh, X. P. and Huang, G. Z. (2008). "Video games in the English Foreign Language Classroom", in Second IEEE International Conference on Digital Games and Intelligent Toys Based Education. Alberta: IEEE, 188-192.

Barab, S., Thomas, M., Dodge, T., Carteaux, R. and Tuzun, H. (2005). "Making learning fun: Quest Atlantis, a game without guns", in Educational Technology Research and Development, 53, 1: 86-107.

Blunt, R. (2009). "Does game-based learning work? Results from three recent studies", in ELearn Magazine. Education and Technology in Perspective, 2009, 12.

Calvo-Ferrer, J. R. (2013). Videojuegos y aprendizaje de segundas lenguas: Análisis del videojuego The Conference Interpreter para la mejora de la competencia terminológica (Doctoral thesis, Universidad de Alicante, Alicante, Spain).

Cohen, J. (1973). "Eta-squared and partial eta-squared in fixed factor ANOVA designs", in Educational and Psychological Measurement, 33: 107-112.

Coller, B. D. and Scott, M. J. (2009). "Effectiveness of using a video game to teach a course in mechanical engineering", in Computers \& Education, 53, 3: 900-912.

Cordova, D. I. and Lepper, M. R. (1996). "Intrinsic motivation and the process of learning: Beneficial effects of contextualization, personalization, and choice", in Journal of Educational Psychology, 88: 715-730.

de Freitas, S. and Oliver, M. (2006). "How can exploratory learning with games and simulations within the curriculum be most effectively evaluated?", in Computers \& Education, 46, 3: 249-264.

Gee, J. P. (2003). What video games have to teach us about learning and literacy. Ney York: Palgrave Macmillan.

Göbel, S., Hardy, S., Wendel, V., Mehm, F. and Steinmetz, R. (2010). "Serious games for health: Personalized exergames", in Proceedings of the international conference on multimedia. New York: ACM, 1663-6.

Guillén-Nieto, V. and Aleson-Carbonell, M. (2012). "Serious games and learning effectiveness: The case of It's a deal!", in Computers \& Education, 58, 1: 435-448.

$\mathrm{Hu}, \mathrm{M}$. M. and Chang, B. (2008). "Massively multiplayer online game supported foreign language listening ability training", in First IEEE International Conference on Digital Games and Intelligent Toys Based Education. Alberta: IEEE, 176-178.

Huyen, N. T. T. and Nga, K. T. T. (2003). "Learning vocabulary through games", in Asian EFL Journal, $5,4$.

Kirriemuir, J. and McFarlane, A. (2004). "Literature review in games and learning", in Future Lab Series Report, 8.

Mayo, M. J. (2007). "Games for science and engineering education", in Communications of the $A C M, 5,7: 31-35$.

Miller, M. and Hegelheimer, V. (2006). "The Sims meet ESL: Incorporating authentic computer simulation games into the language classroom", in Interactive Technology and Smart Education, 3, 4: 311-328.

Moreno-Ger, P., Burgos, D. and Torrente, J. (2009). "Digital games in eLearning environments: Current uses and emerging trends", in Simulation \& Gaming, 40, 5: 669-687.

Nemitcheva, N. (1995). "The psychologist and games in the intensive foreign language gamebased course", in K. Arai \& D. Crookall (eds.), Simulation and gaming across disciplines and cultures. Thousand Oaks, CA: Sage Publications, Inc, 70-74. 
Poplin, A. (2012). "Playful public participation in urban planning: A case study for online serious games", in Computers, Environment and Urban Systems, 36, 3: 195-206.

Rama, P. S., Black, R. W., van Es, E. and Warschauer, M. (2012). "Affordances for second language learning in World of Warcraft", in ReCALL, 24, 3: 322-338.

Rankin, Y., Gold, R. and Gooch, B. (2006). "3D role-playing games as language learning tools", in EUROGRAPHICS 2006, 25, 3.

Ricci, K. E., Salas, E. and Cannon-Bowers, J. A. (1996). "Do computer-based games facilitate knowledge acquisition and retention?", in Military Psychology, 8, 4: 295-307.

Schrader, P. G. and McCreery, M. (2008). "The acquisition of skill and expertise in massively multiplayer online games", in Educational Technology Research and Development, 56, 5.

Schwienhorst, K. (2002). "Why virtual, why environments? Implementing virtual reality concepts in computer-assisted language learning", in Simulation \& Gaming, 33, 2: 196-209.

Wong, W. L., Shen, C., Nocera, L., Carriazo, E., Tang, F., Bugga, S. and Ritterfeld, U. (2007). "Serious video game effectiveness", in Proceedings of the International Conference on Advances in Computer Entertainment Technology. New York: ACM, 49-55.

Yip, F. W. M. and Kwan, A. C. M. (2006). "Online vocabulary games as a tool for teaching and learning English vocabulary", in Educational Media International, 43(3), 233-249. 